\title{
Religion and Self-Regulation
}

\author{
Hui Hou ${ }^{1, a,}$ Benfa Wang, ${ }^{2, b, *}$
}

1Department of education and Psychology, University of Jinan, Jinan, Shandong, China

${ }^{2}$ Department of education and Psychology, University of Jinan, Jinan, Shandong, China

Email : a2641998408@qq.com, bsep_wangbf@ujn.edu.cn

${ }^{*}$ Corresponding author

Key Words: religion, self monitoring, religious image, self-regulation

\begin{abstract}
Previous research has identified the positive correlation between religion and self-regulation. In this paper, progress of current researches concerning the religious self-regulation hypothesis would be introduced and reviewed, from the theoretical explanation, the mode of action and the main research field.Further reveals that the nature of religion may reverse the relationship between religion and self-regulation.
\end{abstract}

\section{宗教对自我调节的影响}

\author{
侯会 ${ }^{1, a}$, , 王本法 $2, b,{ }^{*}$ \\ ${ }^{1}$ 济南大学教育与心理科学学院, 济南, 山东, 中国 \\ ${ }^{2}$ 济南大学教育与心理科学学院, 济南, 山东, 中国 \\ Email : a2641998408@qq.com ,bsep_wangbf@ujn.edu.cn \\ *通讯作者
}

关键词: 自我监测; 宗教意象; 自我调节; 宗教

中文摘要. 以往的研究发现宗教与自我调节间存在显著的相关, 为了进一步揭示两者关系, 本文从理论解释、作用方式和主要研究领域三个方面对宗教影响自我调节的研究进展进行介 绍和评述。并揭示了宗教本质的不同可能反转宗教与自我调节的关系。

\section{1. 概述}

宗教(Religion)指的是与特定的宗教组织或教派归属相联系的信念与行为，其中包括一系 列的教义传统与宗教活动等。宗教要求信徒要遵守宗教准则, 为了维护宗教准则, 个体必须忍 受痛苦或放弃愉快的经历。因此可以预期信徒相对于不信教的个体, 心理幸福感低, 而实证证 据表明相反, 宗教个体内部冲突水平低, 相对于不信教的个体体会到更高水平的积极情绪 (Neyrinck, Vansteenkiste, Lens, Duriez, \& Hutsebaut, 2006;Ryan, Rigby, \& King, 1993; T. B. Smith, McCullough, \&Poll, 2003)。为什么信徒幸福感高，他们的心身更健康?

Koole认为宗教使人们采取更灵活、有效的自我调节。自我调节指控制或改变自己的思维、 情感、冲动以及行为的能力, 尤其是指通过这种改变达到理想、价值观、道德以及社会期望等 标准以逐渐实现自己的长期目标。在这种自我调节下, 信徒可以追求与他们情感需求一致的宗 
教准则, 因此灵活的自我调节过程可以同时满足信徒的宗教准则和情感需求。McCullough （2009）的研究还表明宗教信徒的亲社会行为更高、寿命更长、婚姻更美满。

总之, 信徒的寿命长, 幸福感高, 是因为他们的宗教信仰、行为和认知促进了自我调节 的能力。关于宗教可以有效控制社会的非规范行为和推进社会规范行为在宗教研究史上历史 悠久, 与此相反宗教促进自我调节的研究还没有引起足够的重视。因此本文基于此介绍两者 之间的理论解释、作用方式, 最后介绍了宗教促进自我调节的主要研究领域即宗教可以调节 个体行为和生命意义, 这些是心理健康的基础。所以研究宗教对自我调节的作用有对解释信徒 的寿命长, 幸福感高, 有重要的理论意义。

\section{2. 宗教-自我调节的理论解释}

心理学家认为: 宗教可以促进自我调节, 是因为宗教习俗与信仰利用了与自我调节相似 的心理加工过程。因为这种重叠性, 宗教活动可以促进自我调节技能的获得。此外宗教活动 可以激活认知、动机或情感状态, 提高自我调节的灵活性。总之宗教的核心观念与自我调节 是高度兼容的, 兼容性主要表现在以下几个方面:

\section{1 综合加工过程}

宗教与自我调节兼容的第一种方式在于相互依赖的加工过程。宗教转化可以看成一个加 工过程, 在这个过程中人可以整合过去的经历, 例如在宗教隐喻中神作为一个依恋对象（慈 祥的母亲）, 个人可以将依恋经历和宗教虔诚度整合起来(Granqvist et al., 2010; Kirkpatrick, 1998), 此外象征意义的加工过程需要综合认知能力(Baumann \&Kuhl, 2002) 这种认知风格有 利于自我调节。

\section{2 具身性}

宗教与自我调节兼容的第二种方式在于具身性。许多宗教活动包括了调整身体功能的行 为。例如冥想时控制呼吸, 祈祷时闭上眼睛(Barsalou et al., 2005; Cahn \& Polich, 2006), 这些 身体行为可以帮助个体保持内心的平静。此外身体行为有很深的象征意义, 例如接受葡萄酒 意味着接受基督进入你的生命(Barsalou et al., 2005)。除此之外祈祷时下趾代表着着虔诚(Meier, Hauser, Robinson, Friesen,\& Schjeldahl, 2007)。这些行为可以加深对宗教教义的理解。

\section{3. 宗教-自我调节的作用方式}

自我调节有两个基本因素:目标、自我监测。宗教可以通过影响这些因素进而影响自我调 节，接下来从以下方面介绍宗教对自我调节的作用方式。

\section{1 个体目标}

宗教可以通过影响个体目标，进而影响自我调节。宗教对目标的影响方式有两种，第一 宗教促进目标的整合，从而减少目标之间的冲突。第二宗教影响目标内化的方式。

3.1 .1 宗教减少目标冲突

对个体来说，目标是相互依存，相互影响的。一些目标是一致的，完成其中的一个可以 提高另一个目标实现的可能性, 但目标也可以相互冲突, 追求一个目标的同时, 减少另一个 目标获得的可能性。但宗教可以整合这些目标，使他们之间的冲突最小化。

\section{1 .2 宗教影响目标内化}

宗教影响目标的第二种方式是影响目标的内化方式(Ryan et al., 1993)。Ryan (1993)提出了 两种宗教内化方式: 当人们通过认同来内化一个宗教目标时, 目标是自己选择, 自己完全认 同的。研究表明：相对没有内化宗教目标的个体，内化宗教目标的个体，有更强的自尊心、 人格整合、自我实现倾向，在抑郁、焦虑、躯体化、社会功能障碍方面得分较低。 
相反，当人们通过内投 (把外部对象或自己所赏识的某些人物的特点结合到自己的行为 和信仰中去的一种防御机制）方式来内化宗教目标时, 他们这样做的目的是为了避免焦虑, 内疚或失去尊重。由内投调节的行为会产生更大的冲突, 内部目标只是部分同化。因此, 内 投方式内化宗教目标的个体，有更低的自尊心、人格整合、自我实现倾向，在抑郁、焦虑、 躯体化、社会功能障碍方面得分较高。研究发现: 人们通过认同来内化他们目标的程度与自 我实现呈正相关，而通过内投内化结果相反。

\section{2 自我监测}

自我调节系统必须能够检测系统的当前状态和目标之间的偏差。现代控制理论中，差异 检测装置叫做比较器。比较器对误差的敏感性差异很大。如果误差灵敏度低, 那么一个自我 调节系统可能是（1）响应迟缓（2）在监测差异方面不太准确。自我监测和这种比较过程类 似 (Carver \& Scheier, 1998), 影响自我监测的因素也有力的影响自我调节。

宗教活动可以通过以下方式影响自我监测, 首先宗教环境中的“道德观众” 促进自我监 测。第二宗教仪式激活自我监测。

\subsection{1 宗教环境}

宗教环境是人们根据一定规则评价对方行为的社会环境。相对于其他的社会环境（公园、 街道、工作场合)，相对较少的行为适合宗教环境。Price 和Bouffard (1974)发现, 在15个场 所中 (教堂、休息室、电影院等) 进行 15 种行为（如写作、哭泣、说话等不适当行为）。教 堂被大学生认为是最不合适进行 15 个行为的地方。宗教是高约束的场所, 较少的行为与宗教 环境匹配，其中自我控制行为被认为是最匹配的行为。

因此，我们推测，信仰宗教的人，他们在高约束的宗教环境中呆的时间长，相对非宗教 的个体来说，可能需要花费更多的时间面对评价和 “道德观众” ，因此宗教人士自我控制力 强，有更好的自我监测力，这又反过来导致他们频繁的进行行为调整。

\section{2 .2 宗教仪式激活自我监测}

宗教可以促进自我监测的第二种方式是通过宗教仪式。许多宗教仪式，例如祷告和冥想， 都涉及到将一个人的行为和标准行为进行比较, 例如每周祷告 (天主教徒) 和赎罪日（犹太 人）祷告应该包括对个人的精神和道德缺点进行监测。

有一些证据表明：这种性质的宗教仪式具有预期的效果，Wenger (2007)发现，当他们的 行为没有达到他们的宗教标准, 他们会在宗教领域寻找改善自己的机会。相对于认为达到宗 教标准的个体, 没有达到宗教标准的个体花费更多的时间阅读关于个人宗教成长的文章。这 些研究表明：将宗教标准与宗教领域的实际表现进行比较，促进了自我调节。

\section{4. 宗教一自我调节的主要研究领域}

自我调节成败能解释当今个人和社会上存在的种种问题, 例如个体的健康问题、饮食障 碍、社会上的犯罪行为等方方面面的问题。所以研究自我调节有重要的意义, 而宗教与自我 调节联系紧密。为了进一步验证宗教对自我调节的影响, 研究者做了一系列研究, 其中包括 宗教与行为自我调节和人生意义的自我调节。

\section{1 宗教与行为自我调节}

心理学家把宗教视为一种信仰, 但是宗教也可以促进行为的自我调节(Rueda, Posner, \& Rothbart, 2005)。注意功能影响行为自我调节, 注意起着基础性的作用(Rueda, Posner, \& Rothbart, 2005)。定向注意是实行预期行为所必须的, 并监控行为的结果。此外通过注意力 的分配, 人们可以协调他们的记忆, 思维和情感。因此, 如果宗教能够促进自我调节, 也可 以促进注意功能的提升。 
冥想是宗教传统的一个重要仪式，可以促进注意功能提升（Cahn \& Polich, 2006; McCullough \& Willoughby, 2009)。例如事件相关电位研究表明冥想提高了听觉信号的前注意 加工。其他研究表明冥想扩大了人的注意视野, 培养了与靶向目标快速耦合和瞬脱的能力。

宗教启动的行为效应:宗教习俗与信仰可以引发行为的自我调节。研究发现启动与宗教相 关的概念时, 如神圣, 上帝。在社会交换任务中启动的被试比没启动的被试更加慷慨, 而且 有更多的亲社会行为。

延迟折扣：宗教是否影响有社会意义的行为？宗教对延迟折扣的作用解决了这个问题， 立即满足的冲动可以使人们打折未来的奖励(Metcalfe \& Mischel,1999)。自我调节可以保持未 来奖励的价值来防止这种折扣。和宗教促进自我调节的结论一致, 研究发现宗教个体对未来 奖励的折扣较少(Roelofsma et al., 2009)。值得注意的是：宗教对延迟折扣的影响作用在宗教 信徒参加祈祷活动后变得更强。

\section{2 宗教与人生意义的自我调节}

跨文化研究表明: 宗教能够解决关于人生意义的问题。最近的研究表明内隐自我调节过 程的关键作用在于意义维持机制。如果宗教能促进自我调节, 那么也一定程度上促进人生意 义的调节。

关于自我: 自我是意义的重要来源 (Baumeister, 1987)，不同自我之间的冲突对意义系统 来说是个威胁(McGregor \& Little, 1998)。目前的研究表明: 宗教信仰能从内隐水平上减轻自 我之间的内部冲突, 冥想似乎有可能通过减少自我冲突从而提升人生的意义(McGregor \& Little, 1998)。

人生的意义: 宗教通过使人们远离奢靡, 遵从自己的价值观和自己内心，追求人生的意 义，宗教教义内化的个体感到人生更有意义(Hicks \& King, 2008, )。

恐惧调节与宗教: 关于意义调节的一个有影响力的观点是恐怖调节理论(TMT; Greenberg, Pyszczynski, \& Solomon, 1986)。正如存在主义思想家Kierk认为的那样, 死亡不可避免从而引 起人们的恐惧与焦虑, 为了解除困扰, 人们借助于宗教文化体验超越生命的意义。最近的两 项研究表明: 死亡的提醒增加了基督教徒对宗教的信仰(Norenzayan \& Hansen, 2006), 宗教个 体依靠宗教信仰转移对死亡的关注, 宗教可以帮助信徒超越世俗的生死。内化的宗教信仰是 处理死亡问题的一个重要的资源。

\section{5. 总结与展望}

宗教研究领域广泛、成果众多, 但是都集中于宗教可以有效控制社会的非规范行为和推进 社会规范行为的研究, 宗教对自我调节的促进作用的研究还没有引起足够的关注。而且宗教 与自我调节关系研究证据有限且局限于西方样本, 这种证据的有限性虽然表明了宗教与自我 调整的部分关系, 但这限制了对他们做出进一步推论。除此之外中国是以无神论或非宗教性 为代表的文化, 非宗教信仰是社会人群的主体构成, 因此对于非宗教信仰者精神信仰的研究 具有更为重大的社会意义。

研究还忽略了宗教的本质, 多角度的了解宗教的本质也有助于了解宗教的不利面。有研 究者认为: 宗教的原教旨主义可能会损害宗教对自我调节作用, 因为死板的信念系统抑制了 内隐自我调节过程, 对自我调节和宗教热情的研究发现了这种推理过程的关键性证据。尽管 证据不是很充足, 但当前的发现表明宗教与内隐自我调节的关系可能被宗教原教旨削弱或反 转。

\section{References}

[1] Barsalou, L. W., Barbey, A. K., Simmons, W. K., \& Santos, A.(2005). Embodiment in religious knowledge. Journal of Cognition and Culture, 5, 14-57. 
[2] Baumann, N., \& Kuhl, J. (2002). Intuition, affect, and personality: Unconscious coherence judgments and self-regulation of negative affect. Journal of Personality and Social Psychology, $83,1213-1223$.

[3] Baumeister, R. F. (1987). How the self became a problem: A psychological review of historical research. Journal of Personality and Social Psychology, 52, 163-176.

[4] Cahn, B. R., \& Polich, J. (2006). Meditation states and traits: EEG, ERP, and neuroimaging studies. Psychological Bulletin, 132, 180-211.

[5] Granqvist, P., Mikulincer, M., \& Shaver, P. R. (2010). Religion as attachment: Normative processes and individual differences. Personality and Social Psychology Review, 14, 49-59.

[6] Greenberg, J., Pyszczynski, T., \& Solomon, S. (1986). The causes and consequences of a need for self-esteem: a terror management theory. In R. F. Baumeister (Ed.), Public self and private self (pp. 189-212). New York: Springer-Verlag.

[7] Hicks, J. A., \& King, L. A. (2008). Mood and religion as information about meaning in life. Journal of Research in Personality, 42, 43-57 .

[8] Kadri, N., Tilane, A., El-Batal, M., Taltit, Y., Tahiri, S. M., \& Moussaoui, D. (2000). Irritability during the month of Ramadan. Psychosomatic Medicine, 62, 280-285 .

[9] Kirkpatrick, L. A. (1998). God as a substitute attachment figure: A longitudinal study of adult attachment style and religious change in college students. Personality and Social Psychology Bulletin, 24, 961-973 .

[10]Mccullough, M. E., \& Willoughby, B. L. (2009). Religion, self-regulation, and self-control: associations, explanations, and implications.Psychological Bulletin, 135(1), 69.

[11]McGregor, I., \& Little, B. R. (1998). Personal projects, happiness, and meaning: On doing well and being yourself. Journal of Personality and Social Psychology, 74, 494-512 .

[12]Meier, B. P., Hauser, D. J., Robinson, M. D., Friesen, C. K., \& Schjeldahl, K. (2007). What's 'up' with God? Vertical space as a representation of the divine. Journal of Personality and Social Psychology, 93, 699-710 .

[13]Metcalfe, J., \& Mischel, W. (1999). A hot/cool system analysis of delay of gratification: Dynamics of willpower. Psychological Review, 106, 3-19.

[14]Neyrinck, B., Vansteenkiste, M., Lens, W., Duriez, B., \& Hutsebaut, D. (2006). Cognitive, affective and behavioral correlates of internalization of regulations for religious activities. Motivation and Emotion, 30, 323-334.

[15]Norenzayan, A., \& Hansen, I. G. (2006). Belief in supernatural agents in the face of death. Personality and Social Psychology Bulletin, 32, 174-187.

[16]Ryan, R. M., Rigby, S., \& King, K. (1993). Two types of religious internalization and their relations to religious orientations and mental health. Journal of Personality and Social Psychology, 65, 586-596. 